\title{
Interstitial lung diseases in a lung cancer
} screening trial

\author{
N. Sverzellati*, L. Guerci, G. Randi ${ }^{\#}$, E. Calabrò ${ }^{\dagger}$, C. La Vecchia ${ }^{\#,+}$, A. Marchianò ${ }^{\S}$, \\ A. Pesci ${ }^{f}$, M. Zompatori** and U. Pastorino"
}

ABSTRACT: We assessed the prevalence of interstitial lung disease (ILD) in a cohort of smokers included in a lung cancer screening trial.

Two observers independently reviewed, for the presence of findings consistent with ILD, the computed tomography (CT) examinations of 692 heavy smokers recruited by the Multicentric Italian Lung Detection (MILD) trial. Four CT patterns were considered: usual interstitial pneumonia (UIP), other chronic interstitial pneumonia (OCIP), respiratory bronchiolitis (RB) and indeterminate. Subsequently, the evolution of ILD in those subjects who had undergone a repeat CT examination after 3 yrs was assessed.

The UIP pattern and the OCIP pattern were identified in two (0.3\%) out of 692 and $26(3.8 \%)$ out of 692 patients, respectively; $109(15.7 \%)$ out of 692 patients showed CT abnormalities consistent with RB, while an indeterminate CT pattern was reported in 21 out of $692(3 \%)$ patients. Age, male sex and current smoking status were factors associated with the presence of OCIP and UIP (combined) pattern, although the relationship did not attain statistical significance. A progression of the disease was observed in three (25\%) out of 12 subjects with OCIP who underwent repeat CT after 3 yrs.

Thin-section CT features of ILD, probably representing smoking-related ILD, are not uncommon in a lung cancer screening population and should not be overlooked.

KEYWORDS: Cigarette smoking, computed tomography, interstitial lung disease, lung cancer, screening

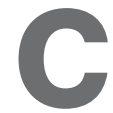
igarette smoking is a recognised risk factor for the development of interstitial lung disease (ILD) [1-3]. The spectrum of thin-section computed tomography (CT) abnormalities associated with cigarette smoking was first defined by REMY-JARDIN and coworkers [4, 5], and since then it has widened continuously. Smokingrelated, thin-section CT abnormalities vary from signs of respiratory bronchiolitis (RB) in asymptomatic patients to severe fibrotic lung disease [1-3], although the mechanisms underlying such associations are not well established. Smoking may cause subclinical parenchymal lung disease consistent with ILD detectable by CT imaging, even among apparently healthy subjects [6].

Although the thin-section CT features of these disorders have been increasingly described in the literature, few data have been reported on their significance in smokers participating in a lung cancer screening trial [7, 8]. A comprehensive assessment of chest CT as a screening tool should include a thorough appreciation of the prevalence and nature of those findings that may have prognostic relevance and require therapeutic interventions.
The purpose of this study was to determine the frequency, appearances and evolution over time of ILD by thin-section CT in a population of smokers included in a lung cancer screening trial.

\section{METHODS}

Data were collected from the Multicentric Italian Lung Detection (MILD) project at the National Cancer Institute (Milan, Italy). The MILD project is an ongoing population-based, randomised controlled lung cancer screening trial, whose primary aim is the assessment of smoking cessation rate among participants, and of the impact of early lung cancer detection on mortality. The MILD project was approved by institutional review boards, and written informed consent was obtained from all participants. For the purpose of this retrospective additional study, and according to the previously obtained Ethics Committee approval and patients' informed consent, we were allowed to use the original data for further research.

Eligibility criteria for the MILD study included age $\geqslant 49$ yrs, current or former smokers (having quit smoking $\leqslant 10$ yrs before recruitment) with at
AFFILIATIONS

${ }^{*}$ Dept of Clinical Sciences, Division of Radiology, University of Parma, Parma,

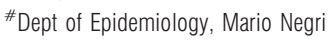
Institute,

Divisions of "Thoracic Surgery, and, ${ }^{\text {s} R a d i o l o g y, ~ N a t i o n a l ~ C a n c e r ~ I n s t i t u t e, ~}$ +Institute of Medical Statistics and Biometry "G.A. Maccacaro", University of Milan, Milan, ${ }^{f}$ Clinical Pulmonology, S. Gerardo Hospital of Monza, University of Milano-Bicocca, Monza, and **Dept of Radiology, S.Orsola Hospital of Bologna, University of Bologna, Bologna, Italy.

\section{CORRESPONDENCE}

N. Sverzellati

Dipartimento di Scienze Cliniche,

Sezione di Radiologia

University di Parma

Ospedale Maggiore di Parma

Padiglione Barbieri

V. Gramsci 14

43100 Parma

Italy

E-mail: nicolasve@tiscali.it

Received:

Dec 232009

Accepted after revision:

Dec 072010

First published online:

Jan 132011 
least 20 pack-yrs of smoking history and no history of cancer within the previous 5 yrs.

Our sampling frame comprised 700 subjects without lung cancer out of the 1,236 subjects recruited between September 2005 and September 2006 (i.e. the first year of recruitment) by MILD. Cases with the longest follow-up CT scan (i.e. after 3 yrs) obtained for the evaluation of lung nodules according to our lung cancer screening protocol were included a priori $(256$ $(20.7 \%)$ out of 1,236 subjects), to allow an assessment of the evolution of any ILD detected to be made [9]. The repeat CT scans were extracted and compared with their corresponding baseline scans. The remaining $444(35.9 \%)$ subjects were randomly selected by the computer and mixed up with the other 256 for review. Such cases had been included in another study addressing a separate hypothesis [10].

Demographic data and smoking history were obtained through a standardised questionnaire for MILD participants. Forced expiratory volume in $1 \mathrm{~s}$ and forced vital capacity expressed as $\%$ predicted were recorded.

\section{Low-dose, thin-section CT protocol}

CT was performed using a 16-detector row CT system (Somatom Sensation 16; Siemens Medical Solutions, Forchheim, Germany). All CT examinations of the whole lung were acquired during one deep inspiratory breath-hold without the use of contrast medium. Standard low-dose CT parameters were as follows: $120 \mathrm{kV}, 30 \mathrm{mAs}, 0.75 \mathrm{~mm}$ collimation, gantry rotation time $0.5 \mathrm{~s}$, pitch 1.5. Visual evaluations of ILD were performed on images reconstructed for detection of pulmonary nodules: 1mm-thick sections with a reconstruction increment of $1 \mathrm{~mm}$ and a sharp kernel (Siemens B50 kernel). All images were viewed at window settings optimised for assessment of lung parenchyma (width 1500-1600 HU; level -500- -600 HU).

\section{Image evaluation}

The CT images of the study population were transferred to a CT workstation (Leonardo; Siemens Medical Solutions) and independently reviewed by two observers (N. Sverzellati and L. Guerci, with 7 and 4 yrs' experience, respectively, in interpreting thin-section CT scans for ILD) for the presence or absence of signs of ILD, without knowledge of the clinical data of the study population. Disagreements on presence variables and on the CT diagnosis were resolved by consensus review of divergent evaluations.
CT examinations were evaluated for the presence of parenchymal changes consistent with ILD, with the specific recommendation to record the presence or absence of ground-glass opacities, consolidation, honeycombing, reticulation and cysts, centrilobular hazy nodules and traction bronchiectasis within areas of ILD. Observers were instructed not to take into account ground-glass opacity when limited to the dependent lung zones (in keeping with dependent atelectasis). Observers were recommended to use the glossary of the Fleischner Society [11].

After assessing the presence of CT findings, the observers evaluated their predominant distribution. The distribution was classified as being predominantly in the upper lung zone (when most of the findings were above the level of the tracheal carina), the lower lung zone (when most of the findings were below the level of the tracheal carina) or diffuse; and as being predominantly peripheral (when findings involved mainly the outer third of the lung), central (when findings were located mainly in the inner two third of the lung) or diffuse. Spatial homogeneity was evaluated through observation of a patchy versus confluent pattern.

Following the initial assessment of the CT findings, each radiologist assessed the $\mathrm{CT}$ patterns for each patient with $\mathrm{CT}$ abnormalities consistent with ILD, with the provision of specific diagnostic criteria based on the current literature and the American Thoracic Society and European Respiratory Society classification for the idiopathic interstitial pneumonias [12, 13]. The CT findings were grouped into three CT patterns as following: 1) usual interstitial pneumonia (UIP)-like pattern; 2) other chronic interstitial pneumonia (OCIP)-like pattern (including either the nonspecific interstitial pneumonia and the desquamative interstitial pneumonia appearances); and 3) RB-like pattern (table 1). When the CT findings did not match any of the criteria, i.e. the abnormalities were equivocal for ILD (e.g. focal ground-glass opacity, unilateral reticulation etc.), the diagnosis was considered as indeterminate.

Subsequently, for each subject with a repeat CT examination after 3 yrs, the observers jointly performed a simultaneous evaluation of the baseline and corresponding follow-up CT examinations to analyse changes over time. On the repeat CT examinations, the extent of CT abnormalities was coded as resolved, similar, greater or lower compared with baseline. The observers also assessed whether the baseline CT pattern showed any change toward another one (e.g. OCIP-like pattern changed into UIP-like pattern).

\section{TABLE 1 Diagnostic criteria for the usual interstitial pneumonia (UIP)-like, other chronic interstitial pneumonia (OCIP)-like,} respiratory bronchiolitis (RB)-like and indeterminate patterns

$\begin{array}{ll}\text { MDCT pattern } & \text { MDCT findings }\end{array}$

UIP-like pattern

OCIP-like pattern

RB-like pattern

Indeterminate pattern
Honeycombing, reticulation, no or minimal ground-glass opacity, peripheral and basal predominance

Predominant ground-glass opacity, no or mild to moderate reticulation, no or traction bronchiectasis, no or minimal honeycombing,

bilateral distribution

Hazy centrilobular nodules, no or mild patches of ground-glass opacities, no or mild reticular abnormalities

Unilateral distribution, predominant consolidation or cysts, and any other finding not included in the UIP-like, OCIP-like and RB-like patterns

MDCT: multidetector computed tomography. 


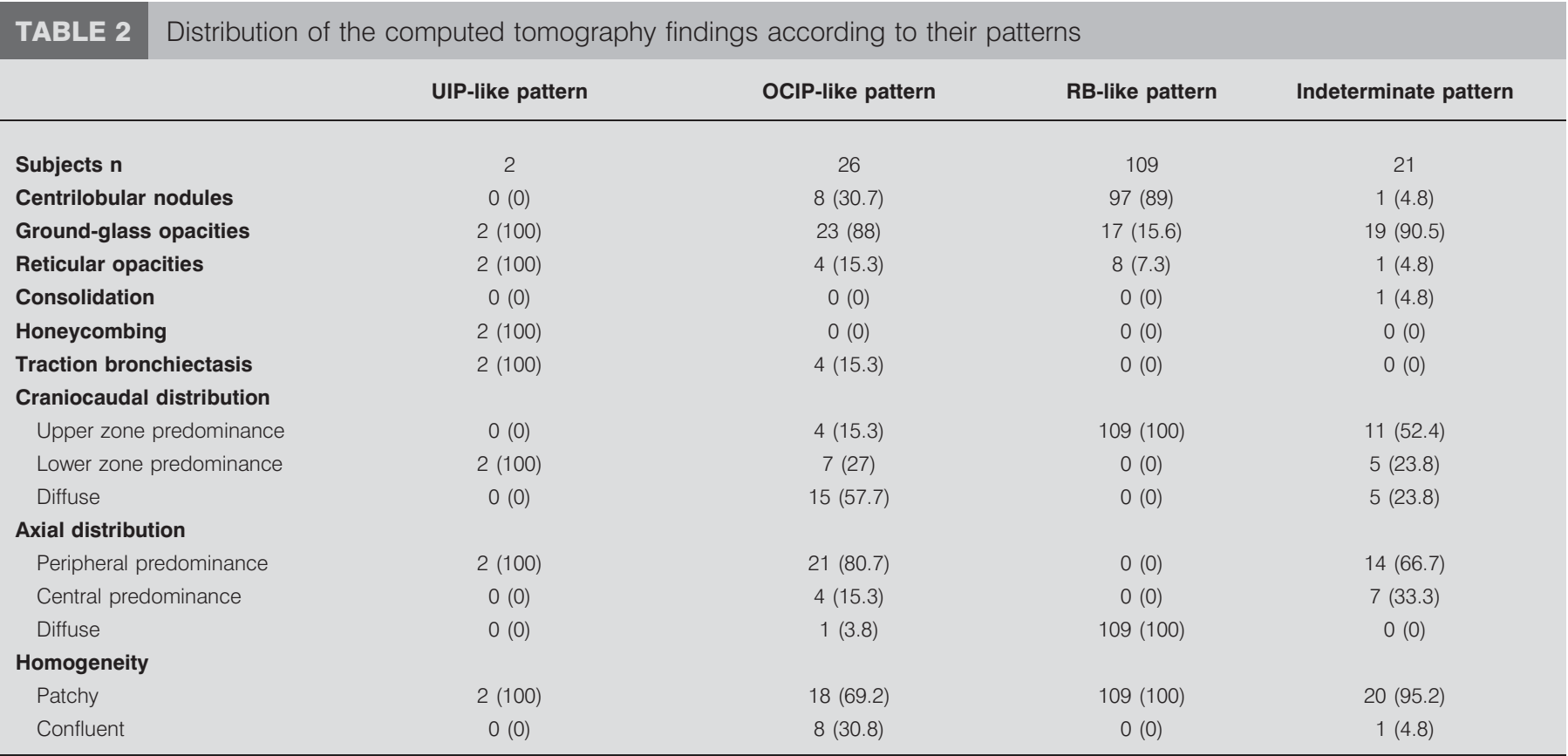

Data are presented as $\mathrm{n}(\%)$, unless otherwise stated. UIP: usual interstitial pneumonia; OCIP: other chronic interstitial pneumonia; RB: respiratory bronchiolitis.

\section{Data analysis}

Unweighted kappa coefficients of agreement $(\kappa)$ were computed for the CT findings and their distribution. Interobserver agreement was classified as poor $(\kappa=0.00-0.20)$, fair $(\kappa=0.21-0.40)$, moderate $(\kappa=0.41-0.60)$, good $(\kappa=0.61-0.80)$ or excellent $(\kappa=0.81-1.00)$ [14].

We estimated ILD prevalence rates by applying a generalised linear regression model of the Poisson family with a logarithmic link function, including adjustment terms of age, sex and smoking status. To further explore ILD prevalence rates, we considered the $\mathrm{CT}$ patterns only among the consecutive cases of our study cohort. Comparative analyses
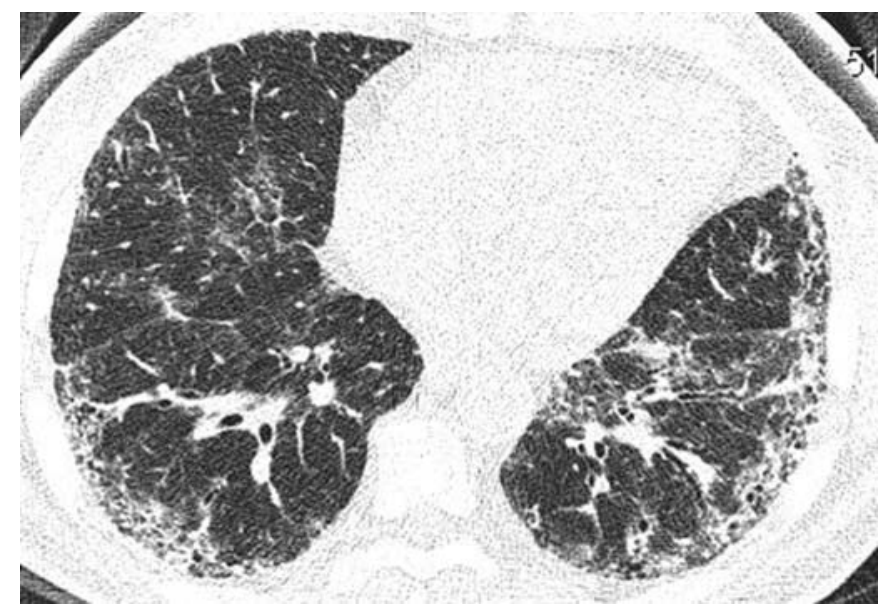

FIGURE 1. A 59-yr-old male with low-dose, thin-section computed tomography (CT) abnormalities resembling a usual interstitial pneumonia-like pattern. Transverse thin-section CT image shows reticular with traction bronchiectasis with minimal honeycombing, predominantly distributed in the subpleural-basal regions. were obtained using both Fisher's exact test and ANOVA to evaluate differences in selected demographic, clinical and pulmonary function test results between subjects with and without ILD.

An unconditional polychotomous logistic regression model was used to assess the association between the presence of the subtypes of ILD and demographic data, clinical features and pulmonary function indices; the corresponding odds ratio (OR) with $95 \%$ confidence interval (CI) were computed. The final model included age (continuous), sex, smoking status (current

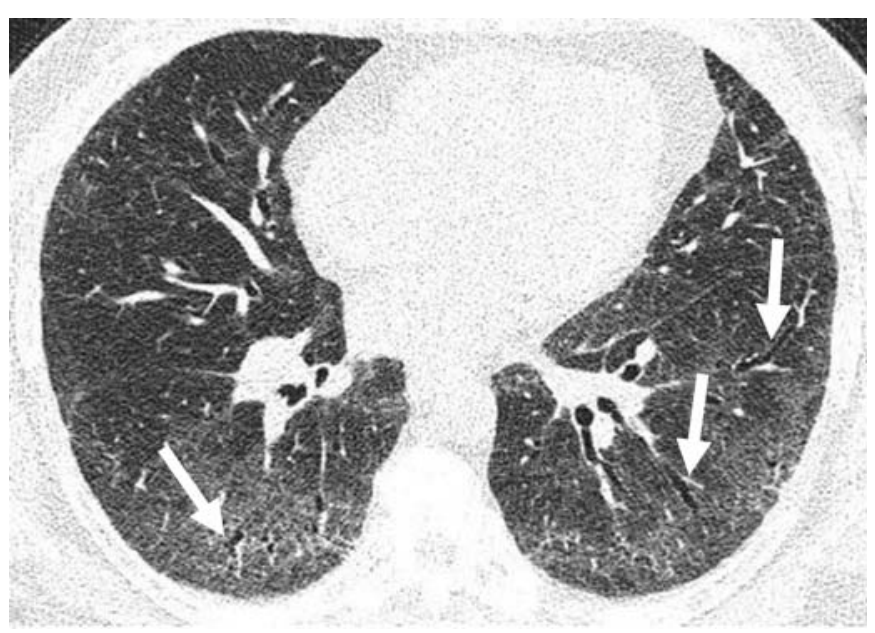

FIGURE 2. Transverse low-dose, thin-section computed tomography (CT) image of a characteristic other chronic interstitial pneumonia-like pattern at the basal regions, in a 64-yr-old female. The CT pattern is made of ground-glass opacities and minimal reticulation with intermingled mild traction bronchiectasis (arrows). 
and former smokers), number of cigarettes per day (continuous) and number of smoking years (continuous). Heterogeneity among ILD subtype risks was assessed using a Wald test with two degrees of freedom [15].

All the analyses were performed with SAS Release 9.1 (SAS Institute Inc., Cary, NC, USA).

\section{RESULTS}

\section{Multidetector CT findings at baseline}

Interobserver agreement was fair $(\kappa=0.3)$ for the diagnosis of the RB-like pattern, moderate $(\kappa=0.6)$ for the OCIP-like pattern, moderate $(\kappa=0.49)$ for the indeterminate pattern and excellent $(\kappa=1.00)$ for the UIP-like pattern. Interobserver agreement was moderate for ground-glass opacity $(\kappa=0.43)$ and centrilobular nodules $(\kappa=0.5)$, good for reticulation $(\kappa=0.62)$ and traction bronchiectasis $(\kappa=0.7)$, and excellent for consolidation $(\kappa=1.0)$ and honeycombing $(\kappa=1.0)$. Interobserver agreement was
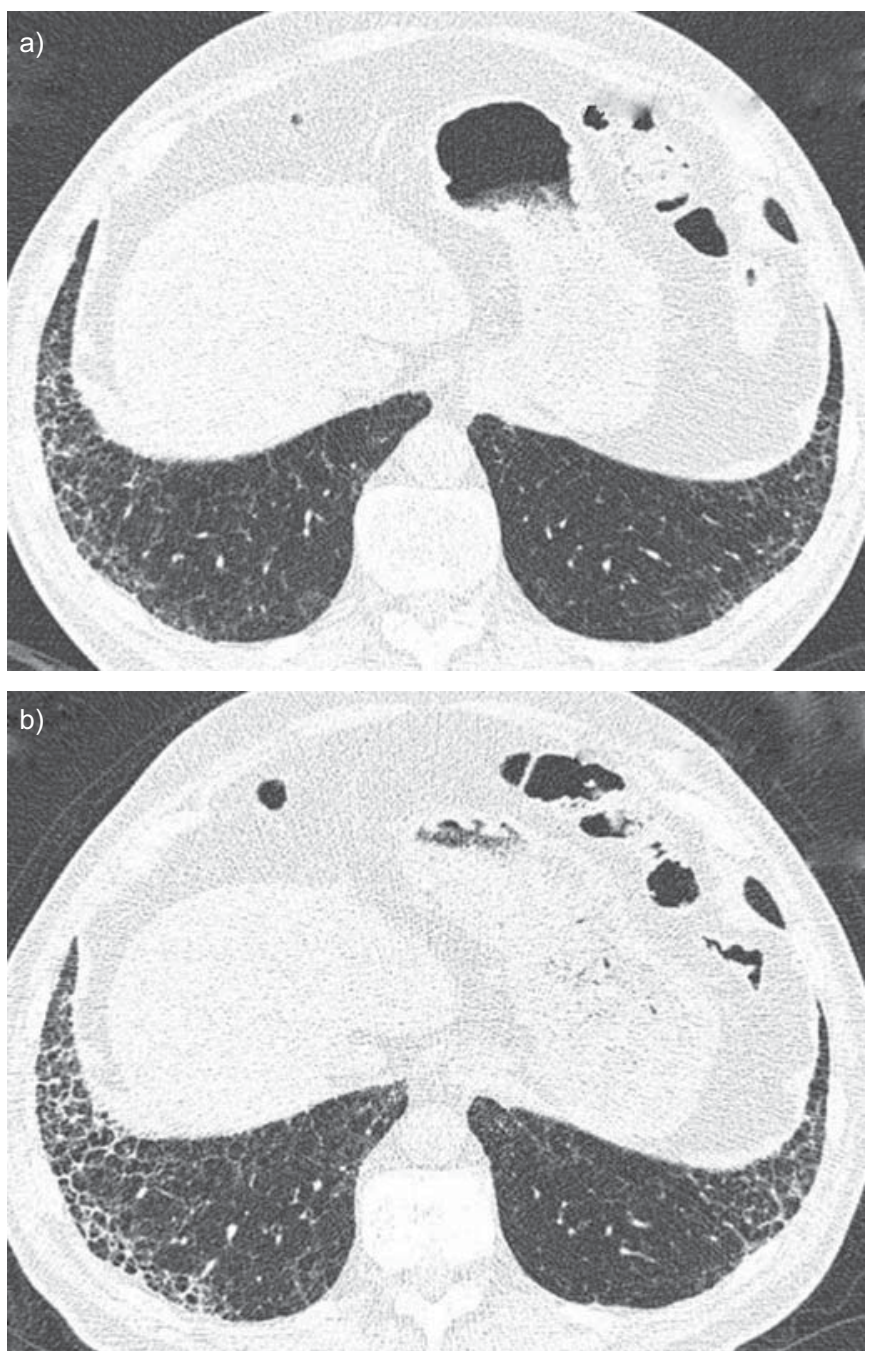

FIGURE 3. a) A 52-yr-old male with low-dose, thin-section computed tomography (CT) findings regarded as other chronic interstitial pneumonia-like pattern by both the observers. Note the evident reticular opacities at the level of the basal-subpleural regions. b) Repeat CT examination 3 yrs later showing an increase in extent of reticulation with developed honeycombing regarded as usual interstitial pneumonia-like pattern by both the observers. moderate for both upper predominant $(\kappa=0.45)$ and lower predominant $(\kappa=0.45)$ distribution as well as moderate for both peripheral $(\kappa=0.5)$ and central $(\kappa=0.45)$ distribution; and it was fair for patchy $(\kappa=0.4)$, diffuse $(\kappa=0.3)$ and confluent $(\kappa=0.3)$ distribution.

Eight CT examinations were not considered eligible for the visual score due to motion artefacts, and the final study cohort thus comprised 692 subjects.

At baseline, the UIP-like pattern and the OCIP-like pattern were identified in two $(0.3 \%)$ out of 692 and $26(3.8 \%)$ out of 692 patients, respectively; the crude prevalence of the combined UIP/OCIP pattern was $4.0 \%$ (95\% CI 2.8-5.9\%), which increased to $9.3 \%(95 \%$ CI $5.2 \%-16.7 \%)$ after adjustment for age, sex and smoking status. A total of 109 (15.7\%) out of 692 patients showed CT abnormalities consistent with RB, while $\mathrm{CT}$ abnormalities equivocal for ILD were reported in $21(3 \%)$ out of 692 subjects. CT examinations were regarded as normal in $534(77.2 \%)$ out of 692 subjects.

Considering only consecutive cases (420 (61\%) out of 692$)$, the UIP-like pattern was identified in one $(0.2 \%)$ out of 420 , the OCIP-like pattern in $14(3.3 \%)$ out of 420 , the RB-like pattern in $69(16.4 \%)$ out of 420 , and the indeterminate pattern in $14(3.3 \%)$ out of 420 . The crude prevalence of the combined UIP/OCIP pattern among consecutive cases was 3.6\% (95\% CI $2.1 \%-5.9 \%$ ), which increased to $10.1 \%$ (95\% CI $4.8 \%-21.1 \%$ ) after adjustment for age, sex and smoking status.

Detailed descriptions of CT findings are summarised in table 2. The presence of subpleural basal honeycombing with peripheral reticular opacities and traction bronchiectasis was regarded as consistent with a UIP-like pattern in two $(0.3 \%)$ out of 692 cases (fig. 1). These two subjects were current smokers with a long history of smoking (40 and $50 \mathrm{yrs,}$ respectively).

Peripheral ground-glass opacity was the most frequent finding in subjects with a OCIP-like pattern (23 (88\%) out of 26$)$, while reticular opacities were recorded in four (15.3\%) out of 26 cases (figs 2 and 3). In eight (30.8\%) out of 26 cases, a OCIP-like

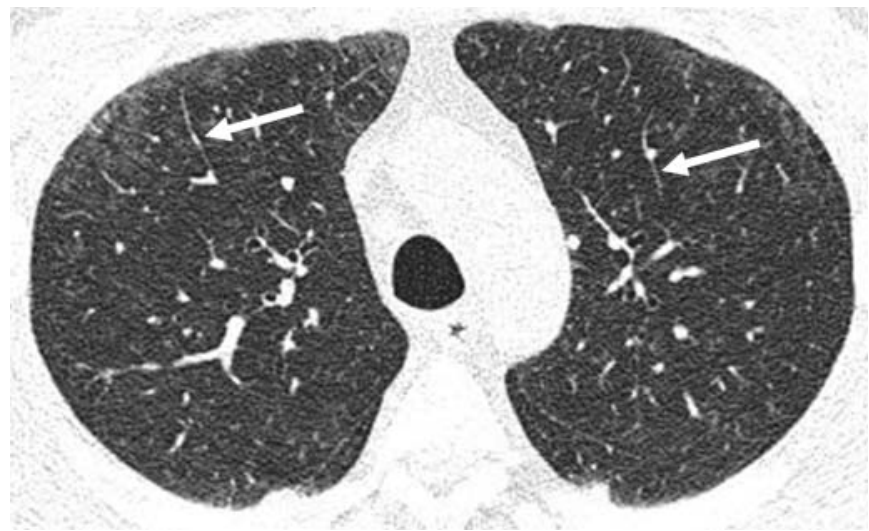

FIGURE 4. A respiratory bronchiolitis-like pattern in a 56-yr-old female. Lowdose, thin-section computed tomography image shows peripheral ground-glass opacity in the ventral regions, centrilobular hazy nodules, scarce interlobular septal thickening (arrows) and mild paraseptal emphysema. 

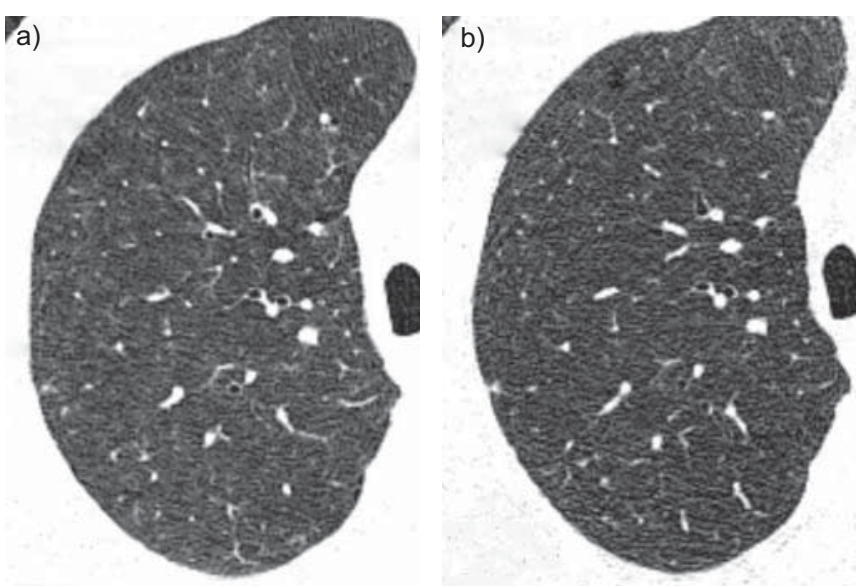

FIGURE 5. Thin-section computed tomography (CT) images in a 64-yr-old quitter. a) Thin-section CT image targeted to the right lung at baseline shows faint centrilobular micronodules. b) 3 yrs later, a decrease in profusion of the nodules was evident at repeat CT.

pattern coexisted with hazy centrilobular nodules predominating in the upper zones. Small cysts intermingled with peripheral ground-glass opacity were observed in one case out of 26. Four $(15.3 \%)$ out of 26 cases with a OCIP-like pattern showed traction bronchiectasis.

Diffuse hazy centrilobular nodules predominating in the upper lung zone were the most frequent finding (97 (89\%) out of 109) in patients with a RB-like pattern. In subjects with a RB-like pattern, patchy ground-glass opacity associated with centrilobular nodules was reported in 17 out of 109 cases (figs 4 and 5), while ground-glass opacity was identified either as an isolated finding or associated with scarce interlobular septal thickening in eight out of 109 and four out of 109 cases, respectively. Overall, mild interlobular septal thickening was reported in eight out of 109 cases with an RB-like pattern.

Most (19 (90.5\%) out of 21$)$ of the indeterminate CT patterns were characterised predominantly by very mild patchy ground-glass opacities (fig. 6). In three out of 19 cases, ground-glass opacity was unilateral, while in one out of 19 it was limited to the middle lobe and the lingula. Two out of 21 cases showed indistinct (acinar) ground-glass opacities predominating in the upper lung zone, while another showed basal bilateral consolidation.

\section{Demographic, clinical and functional findings at baseline}

Demographic and smoking history, and pulmonary function data, are shown according to the presence of ILD in table 3. Because of the small number of cases with a UIP-like pattern, the UIP-like cases were grouped with the OCIP ones for comparison analyses. Subjects with CT abnormalities either consistent with or equivocal for ILD showed a longer history of smoking and were more frequently current smokers. Both subjects with a UIP/OCIP-like pattern and those with an indeterminate CT pattern were older than smokers without signs of ILD. According to the multivariate polychotomous logistic regression model (table 4), the risk for the presence of UIP/OCIP-like patterns was directly associated with age (OR 2.06 for 10 yrs (95\% CI 0.94-4.51)), male sex (OR 2.71 (95\%

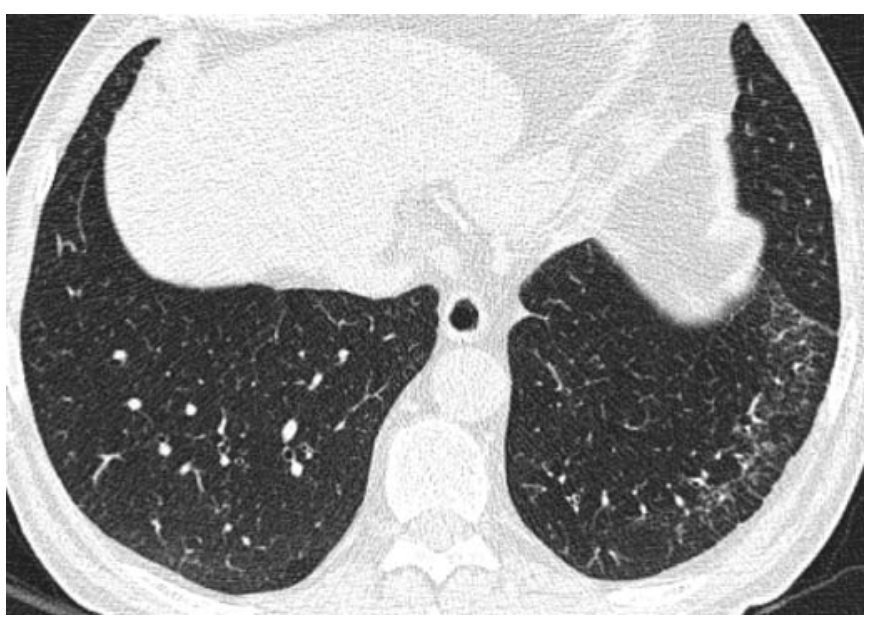

FIGURE 6. A 68-yr-old male with findings suggestive of interstitial lung disease, classified as indeterminate pattern on computed tomography by both the observers. Minimal ground-glass opacities are limited to the subpleural-basal regions of the left lung.

CI 0.90-8.15)) and current smoking status (OR 2.29 (95\% CI 0.80-6.60)), whereas the RB-like pattern was inversely associated with age (OR 0.51 for 10 yrs (95\% CI $0.32-0.84)$ ) and directly associated with current smoking status (OR 3.35 (95\% CI 1.70-6.58)). Heterogeneity between the RB-like pattern risk and the UIP/OCIP-like pattern risk was observed for age $(p=0.002)$, but not for smoking status $(p=0.545)$.

\section{Evolution between baseline and follow-up multidetector CT findings and smoking status}

There were 256 multidetector CT scans after 3 yrs, with 44 $(17.1 \%), 13(5 \%)$ and $12(4.7 \%)$ of them having a RB-like pattern, an indeterminate pattern and a OCIP-like pattern on baseline CT scan, respectively. None of the subjects with a UIP-like pattern at baseline underwent a CT examination after 3 yrs.

Follow-up CT scan of one subject with an OCIP-like pattern at baseline was interpreted as being consistent with UIP (fig. 3b), while two other cases with an OCIP-like pattern showed an increase in extent of the reticular abnormalities (three $(25 \%)$ out of 12 ). The rest (nine (75\%) out of 12 ) of the repeat CT examinations of the subjects with an OCIP-like pattern did not show any changes in either the pattern or extent of the disease.

Among the 13 subjects with an indeterminate CT pattern, the CT findings were similar in eight out of 13 cases and resolved in three out of 13 cases after 3 yrs. The extent of ground-glass opacity increased in two out of 13 cases.

No RB-like patterns changed their appearance. Among the 42 subjects with centrilobular nodules at baseline, nodules were similar in $36(85.7 \%)$ out of 42 , while they increased in profusion in three out of 42 cases, decreased in one out of 42 cases (fig. 5b), and completely resolved in one out of 42 cases. Among the nine subjects with ground-glass opacities at baseline, the extent of ground-glass opacities was similar in seven out of nine cases, decreased in one case and increased in one case out of nine, respectively. Ground-glass opacities superimposed ex novo on centrilobular nodules in only one case. One patient who had 
TABLE 3 Demographic and clinical characteristics of 692 subjects according to the presence of thin-section computed tomography patterns

\begin{tabular}{|c|c|c|c|c|c|}
\hline & RB-like pattern & $\begin{array}{l}\text { OCIP- and UIP-like } \\
\text { patterns }\end{array}$ & Indeterminate pattern & Normal & p-value ${ }^{\#}$ \\
\hline Subjects $n$ & 109 & 28 & 21 & 534 & \\
\hline Age yrs & $55.5 \pm 5.4$ & $60.3 \pm 6.5$ & $58.1 \pm 7.1$ & $57.2 \pm 5.9$ & 0.001 \\
\hline \multicolumn{6}{|l|}{ Sex } \\
\hline Female & $36(33.0)$ & $4(14.3)$ & $4(19.1)$ & $185(34.6)$ & \\
\hline Former & $11(10.1)$ & $5(18.5)$ & $3(14.3)$ & $159(29.8)$ & \\
\hline Current & $98(89.9)$ & $22(81.5)$ & $18(85.7)$ & $375(70.2)$ & $<0.001$ \\
\hline \multicolumn{6}{|c|}{ Number of cigarettes per day } \\
\hline$<20$ & $24(22.0)$ & $8(28.6)$ & $5(23.8)$ & $122(22.9)$ & \\
\hline 20 & $46(42.2)$ & $7(25.0)$ & $10(47.6)$ & $230(43.1)$ & \\
\hline$>20$ & $39(35.8)$ & $13(46.4)$ & $6(28.6)$ & $182(34.1)$ & 0.633 \\
\hline$\geqslant 40$ & $48(44.0)$ & $15(53.6)$ & $10(47.6)$ & $226(42.3)$ & 0.343 \\
\hline Yrs of stopping smoking & $3.8 \pm 3.1$ & $3.8 \pm 3.3$ & $3.5 \pm 2.1$ & $5.2 \pm 3.3$ & 0.438 \\
\hline FEV $_{1} \%$ pred & $93.0 \pm 18.8$ & $89.0 \pm 17.3$ & $98.0 \pm 18.7$ & $95.3 \pm 20.6$ & 0.317 \\
\hline FVC \% pred & $101.4 \pm 21.0$ & $100.8 \pm 18.4$ & $106.9 \pm 18.7$ & $103.8 \pm 21.4$ & 0.574 \\
\hline
\end{tabular}

Data are presented as mean \pm SD or $n(\%)$, unless otherwise stated. RB: respiratory bronchiolitis; OCIP: other chronic interstitial pneumonia; UIP: usual interstitial pneumonia; FEV1: forced expiratory volume in $1 \mathrm{~s} ; \%$ pred: \% predicted; FVC: forced vital capacity. ${ }^{*}$ : derived from Fisher's exact test for categorical variables and ANOVA analysis for continuous variables.

mild interlobular septal thickening at baseline did not show any change in extent of those abnormalities at follow-up CT.

In two out of 184 smokers without any sign of ILD at baseline, diffuse peripheral ground-glass opacity resembling an OCIPlike pattern and centrilobular hazy nodules predominating in the upper zones were respectively reported. Both of them were persistent former smokers.

As detailed in table 5, the frequency of quitters among subjects with either an OCIP-like or an indeterminate CT pattern was greater than that of those with a RB-like pattern and controls.
All subjects with either an OCIP-like, an RB-like or an indeterminate pattern showing an increase in the disease extent at repeat CT were persistent current smokers, whereas those in whom the disease extent decreased were quitters.

\section{DISCUSSION}

This study shows that important abnormalities consistent with fibrotic ILD may be encountered in participants in a lung cancer screening trial. In our study population, the crude prevalence of UIP/OCIP was $4.0 \%$ (95\% CI $2.8 \%-5.9 \%)$, while the prevalence adjusted for age, sex and smoking status was

TABLE 4 Interstitial lung disease for selected characteristics by computed tomography patterns

\begin{tabular}{|c|c|c|c|}
\hline & RB-like pattern & OCIP- and UIP-like patterns & p Heterogeneity \\
\hline Age (10 yrs) & $0.51(0.32-0.84)$ & $2.06(0.94-4.51)$ & 0.002 \\
\hline Female & 1 & 1 & \\
\hline Male & $1.12(0.70-1.77)$ & $2.71(0.90-8.15)$ & 0.136 \\
\hline \multicolumn{4}{|l|}{ Smoking status } \\
\hline Number of cigarettes day ${ }^{-1}$ (10 units) & $1.10(0.89-1.37)$ & $1.18(0.81-1.73)$ & 0.734 \\
\hline Number of smoking yrs (10 yrs) & $1.36(0.91-2.04)$ & $1.17(0.60-2.26)$ & 0.687 \\
\hline
\end{tabular}




\begin{tabular}{|c|c|c|c|c|}
\hline Stable & 31 & 6 & 1 & \\
\hline Increased & 3 & 0 & 0 & \\
\hline Decreased & 0 & 0 & 3 & \\
\hline Stable & 4 & 2 & 3 & \\
\hline Increased & 3 & 0 & 0 & \\
\hline Decreased & 0 & 0 & 0 & \\
\hline Indeterminate pattern & $7(53.9)$ & $2(15.4)$ & $4(30.8)$ & 0 \\
\hline Stable & 6 & 2 & 1 & \\
\hline Increased & 1 & 0 & 0 & \\
\hline
\end{tabular}

Data are presented as $n$ (\%) or $\mathrm{n}$. RB: respiratory bronchiolitis; OCIP: other chronic interstitial pneumonia. ${ }^{*}$ : ex-smokers who resumed smoking between baseline and repeat computed tomography examinations.

9.3\% (95\% CI 5.2\%-16.7\%). Importantly, such prevalence data were similar to those obtained by limiting the evaluation only to the consecutive cases of our study cohort. This prevalence is between that reported by other studies evaluating the incidental findings in lung cancer screening. In the DutchBelgian Randomised Controlled Lung Cancer Screening Trial (NELSON study) [7], signs of pulmonary fibrosis were reported in $117(8 \%)$ out of 1,409 cases, whereas they were observed in six (1.3\%) out of 449 cases recruited by the ProActive Lung Cancer Detection (PALCAD) study [8].

Another recent study has shown by means of CT densitometric techniques in a large population-based cohort of smokers that abnormalities consistent with ILD are frequently detectable in asymptomatic smokers and cumulative and current cigarette smoking were both independently associated with them [6]. However, an advantage of our study was to assess the CT pattern among subjects with ILD to improve the discriminatory information provided by CT. To our knowledge, this is the first study that has addressed such evaluation in a lung cancer screening population, and it was useful to further elucidate the prevalence of the ILD in a large population of asymptomatic smokers and to explore the relevance of these CT features from a prognostic standpoint.

The findings that two (0.3\%) out of 692 cases showed a UIP-like CT pattern at baseline and another with a OCIP-like pattern came to resemble that of UIP after 3 yrs, highlights the importance of not overlooking the presence of ILD in a lung cancer screening population. A growing body of studies shows that smoking is an independent risk factor for the development of UIP, which, in the majority of cases, carries a prognosis worse than that of many lung cancers [16-18]. Notably, the relatively low prevalence of the UIP-like CT pattern $(0.4 \%$ including the case detected at repeat CT) in our study cohort was slightly lower than that of the lung cancers detected by our screening project in the first year of recruitment $(10(0.8 \%)$ out of 1,236$)$.

We reported $26(3.7 \%)$ out of 705 cases with an OCIP-like pattern, mainly characterised by peripheral ground-glass opacities. The coexistence of hazy centrilobular nodules in about one-third of these cases further emphasises the overlap between the ILD features related to smoking. The reported association between the CT abnormalities of the UIP/OCIPlike pattern and smoking status, with current smokers more at risk of developing such diseases, further corroborates the link with smoking; such a link is also supported by the longitudinal behaviour of the OCIP-like pattern, with disease progression observed among persistent current smokers and decrease in quitters. By contrast, the finding that the presence of a UIP/ OCIP-like pattern was associated with age is in keeping with recent observations of ILD findings frequently seen among asymptomatic elderly individuals independent of smoking history [19]. Of note, males were more at risk for the UIP/OCIP pattern, in keeping with previous findings showing such changes more often in male than female smokers $[17,20]$.

However, the relevance of such multidetector CT abnormalities remains open to debate as $25 \%$ of the OCIP cases in our study showed a progression after 3 yrs, suggesting that attention should be paid even to these more apparently indolent ILDs to prevent fibrosis progression or the occurrence of complications. However, it appears possible that abnormalities defining the OCIP-like pattern might be symptomatic as shown by the relatively higher percentages of former smokers at follow-up among subjects with an OCIP-like pattern as compared to either controls and those with RB.

Although RB is seen as a very common histological finding in cigarette smokers, and of little clinical significance, we have undertaken the evaluation of CT signs of RB to extend the knowledge on their prevalence and relationship with smoking 
history in a large study population. In our study, the prevalence of CT changes consistent with RB was lower than those reported previously. Whilst REMY-JARDIN et al. [5] found upper zone-predominant micronodules in $26(27 \%)$ out of 98 asymptomatic smokers, we found signs of RB in $109(15.7 \%)$ out of 692 smokers despite the fact that individuals recruited by MILD were heavier smokers. Such a discrepancy may have several explanations: in particular, the use of a low-dose CT protocol in our lung cancer screening trial may have lowered the detection rate of hazy nodules and ground-glass opacity [21]. Furthermore, in contrast with another study of REMY-JARDIN et al. [22], which showed changes of centrilobular nodules over a mean period of $5.5 \mathrm{yrs}$ in $11(64.7 \%)$ out of 17 smokers, only five (12\%) out of 42 cases with centrilobular nodules displayed changes after 3 yrs in our study. Notably, in our study, no RBlike pattern changed toward another one. However, the different time windows might certainly explain the differences with the study of REMY-JARDIN et al. [22]. In keeping with the study of FRAIG et al. [23], we showed that current smokers were more at risk of showing RB compared with former smokers, and RB findings may persist after smoking cessation, being present in both former and quitting smokers. The finding that the RBlike pattern was inversely associated with age is in keeping with previous observations showing $\mathrm{RB}$ changes occurring more frequently in young current smokers [24, 25].

Our study does have some limitations. The inclusion a priori of a sub-group of subjects having follow-up CT examination at the longest available time frame might have biased our observations. Nevertheless, we would like to stress that such a bias is likely to be negligible in the absence of any reason why individuals with a lung nodule should be more or less predisposed to developing ILD. The lack of histological data and a full clinical evaluation in the lung cancer screening setting induced speculation to attempt a more complete characterisation of the abnormalities consistent with or suspicious for ILD. Similarly to the scheme proposed by CHURG and MÜLLER [12], the CT findings were grouped into three fundamental CT patterns (i.e. UIP-like, OCIP-like and RB-like patterns) that generally carry different prognoses. However, a significant radiological overlap exists among those $\mathrm{CT}$ patterns. While the typical thin-section CT pattern is highly predictive of a histological diagnosis of UIP, the OCIP-like CT pattern may correspond to a wider spectrum of histological diagnoses such as desquamative interstitial pneumonia (DIP) (the case with some cysts intermingled with ground-glass opacities was in keeping with DIP) and even UIP (this might fit with our case showing OCIP-like pattern at baseline that changed the CT appearance into a UIP-like pattern after 3 yrs) [26-29]. The RB-like pattern was assumed to comprise both pure RB and RB-ILD, as they usually display very similar CT pattern and cannot be differentiated solely from the nature of CT abnormalities. Thus such distinction is possible only on the basis of a careful clinical evaluation that was not part of our lung cancer screening protocol. Furthermore, attempting an interpretation of the indeterminate $\mathrm{CT}$ patterns, as well as explaining the two normal cases who developed signs of ILD at repeat CT examination, would be very dangerous. The lack of prone images was a limitation because it is possible that some cases with true ground-glass opacity limited to dependent lung zones was not taken into account, reducing the estimation of the ILD prevalence. Of note, no CT signs of Langerhans' cell histiocytosis were observed among the indeterminate CT patterns. Finally, we decided to evaluate only the repeat CT examinations after 3 yrs (i.e. the longest follow-up available in the MILD trial) as such a time window was assumed to be sufficient for a proper observation of the evolution of the ILD abnormalities; however, a longer follow-up is probably needed, particularly to evaluate the OCIP-like patterns. It has to be emphasised that the assessment of early ILD over large study populations like those of lung cancer screening trials is challenging and time-consuming. The new system of visual CT scoring proposed by WASHKO et al. [30] for the identification of changes suggestive of early ILD is intriguing as it involves a reduction of more than half in the number of chest $\mathrm{CT}$ evaluations compared to a consensus reading.

In conclusion, our study suggests that CT signs of ILD are not uncommon in a lung cancer screening cohort and attention should be paid to not overlooking progressive fibrosis.

\section{STATEMENT OF INTEREST}

None declared.

\section{REFERENCES}

1 Wells AU, Nicholson AG, Hansell DM. Challenges in pulmonary fibrosis. 4: smoking-induced diffuse interstitial lung diseases. Thorax 2007; 62: 904-910.

2 Attili AK, Kazerooni EA, Gross BH, et al. Smoking-related interstitial lung disease: radiologic-clinical-pathologic correlation. Radiographics 2008; 28: 1383-1396.

3 Vassallo R, Ryu JH. Tobacco smoke-related diffuse lung diseases. Semin Respir Crit Care Med 2008; 29: 643-650.

4 Remy-Jardin M, Remy J, Gosselin B, et al. Lung parenchymal changes secondary to cigarette smoking: pathologic-CT correlations. Radiology 1993; 186: 643-651.

5 Remy-Jardin M, Remy J, Boulenguez C, et al. Morphologic effects of cigarette smoking on airways and pulmonary parenchyma in healthy adult volunteers: CT evaluation and correlation with pulmonary function tests. Radiology 1993; 186: 107-115.

6 Lederer DJ, Enright PL, Kawut SM, et al. Cigarette smoking is associated with subclinical parenchymal lung disease: the MultiEthnic Study of Atherosclerosis (MESA)-lung study. Am J Respir Crit Care Med 2009; 180: 407-414.

7 van de Wiel JC, Wang Y, Xu DM, et al. Neglectable benefit of searching for incidental findings in the Dutch-Belgian lung cancer screening trial (NELSON) using low-dose multidetector CT. Eur Radiol 2007; 17: 1474-1482.

8 MacRedmond R, Logan PM, Lee M, et al. Screening for lung cancer using low dose CT scanning. Thorax 2004; 59: 237-241.

9 Marchianò A, Calabrò E, Civelli E, et al. Pulmonary nodules: volume repeatability at multidetector CT lung cancer screening. Radiology 2009; 251: 919-925.

10 Sverzellati N, Calabrò E, Randi G, et al. Sex differences in emphysema phenotype in smokers without airflow obstruction. Eur Respir J 2009; 33: 1320-1328.

11 Hansell DM, Bankier AA, MacMahon H, et al. Fleischner Society: glossary of terms for thoracic imaging. Radiology 2008; 246: 697-722.

12 Churg A, Müller NL. Cellular vs fibrosing interstitial pneumonias and prognosis: a practical classification of the idiopathic interstitial pneumonias and pathologically/radiologically similar conditions. Chest 2006; 130: 1566-1570.

13 American Thoracic Society, European Respiratory Society. American Thoracic Society/European Respiratory Society International 
Multidisciplinary Consensus Classification of the Idiopathic Interstitial Pneumonias. Am J Respir Crit Care Med 2002; 165: 277-304.

14 Coblentz CL, Babcook CJ, Alton D, et al. Observer variation in detecting the radiologic features associated with bronchiolitis. Invest Radiol 1991; 26: 115-118.

15 Hosmer DW, Lemeshow S, eds. Applied Logistic Regression. 2nd Edn. New York, John Wiley \& Sons Inc, 2000.

16 Baumgartner KB, Samet JM, Stidley CA, et al. Cigarette smoking: a risk factor for idiopathic pulmonary fibrosis. Am J Respir Crit Care Med 1997; 155: 242-248.

17 Antoniou KM, Hansell DM, Rubens MB, et al. Idiopathic pulmonary fibrosis: outcome in relation to smoking status. Am J Respir Crit Care Med 2008; 177: 190-194.

18 Maher TM, Wells AU, Laurent GJ. Idiopathic pulmonary fibrosis: multiple causes and multiple mechanisms? Eur Respir J 2007; 30: 835-839.

19 Copley SJ, Wells AU, Hawtin KE, et al. Lung morphology in the elderly: comparative CT study of subjects over 75 years old versus those under 55 years old. Radiology 2009; 251: 566-573.

20 Marten K, Milne D, Antoniou KM, et al. Non-specific interstitial pneumonia in cigarette smokers: a CT study. Eur Radiol 2009; 19 : 1679-1685.

21 Zwirewich CV, Mayo JR, Müller NL. Low-dose high-resolution CT of lung parenchyma. Radiology 1991; 180: 413-417.

22 Remy-Jardin M, Edme JL, Boulenguez C, et al. Longitudinal follow-up study of smoker's lung with thin-section CT in correlation with pulmonary function tests. Radiology 2002; 222: 261-270.
23 Fraig M, Shreesha U, Savici D, et al. Respiratory bronchiolitis: a clinicopathologic study in current smokers, ex-smokers, and never-smokers. Am J Surg Pathol 2002; 26: 647-653.

24 Niewoehner DE, Kleinerman J, Rice DB. Pathologic changes in the peripheral airways of young cigarette smokers. N Engl J Med 1974; 291: 755-758.

25 Moon J, du Bois RM, Colby TV, et al. Clinical significance of respiratory bronchiolitis on open lung biopsy and its relationship to smoking related interstitial lung disease. Thorax 1999; 54: 1009-1014.

26 Kligerman SJ, Groshong S, Brown KK, et al. Nonspecific interstitial pneumonia: radiologic, clinical, and pathologic considerations. Radiographics 2009; 29: 73-87.

27 Flaherty KR, Thwaite EL, Kazerooni EA, et al. Radiological versus histological diagnosis in UIP and NSIP: survival implications. Thorax 2003; 58: 143-148.

28 Vassallo R, Jensen EA, Colby TV, et al. The overlap between respiratory bronchiolitis and desquamative interstitial pneumonia in pulmonary Langerhans cell histiocytosis: high-resolution CT, histologic, and functional correlations. Chest 2003; 124: 1199-1205.

29 Silva CI, Müller NL, Hansell DM, et al. Nonspecific interstitial pneumonia and idiopathic pulmonary fibrosis: changes in pattern and distribution of disease over time. Radiology 2008; 247: 251-259.

30 Washko GR, Lynch DA, Matsuoka S, et al. Identification of early interstitial lung disease in smokers from the COPDGene Study. Acad Radiol 2010; 17: 48-53. 